\title{
EchoGéo
}

34 | 2015

Varia

\section{Premières réflexions sur la préfiguration d'un observatoire lyonnais du climat}

Didier Soto, Florent Renard et Emmanuel Thimonier-Rouzet

\section{OpenEdition}

\section{Journals}

Édition électronique

URL : https://journals.openedition.org/echogeo/14377

DOI : 10.4000/echogeo. 14377

ISSN : 1963-1197

\section{Éditeur}

Pôle de recherche pour l'organisation et la diffusion de l'information géographique (CNRS UMR 8586)

Référence électronique

Didier Soto, Florent Renard et Emmanuel Thimonier-Rouzet, « Premières réflexions sur la préfiguration d'un observatoire lyonnais du climat », EchoGéo [En ligne], 34 | 2015, mis en ligne le 15 décembre 2015, consulté le 01 août 2021. URL : http://journals.openedition.org/echogeo/14377 ; DOI : https://doi.org/ 10.4000/echogeo.14377

\section{Ce document a été généré automatiquement le 1 août 2021.}

EchoGéo est mis à disposition selon les termes de la licence Creative Commons Attribution - Pas d'Utilisation Commerciale - Pas de Modification 4.0 International (CC BY-NC-ND) 


\title{
Premières réflexions sur la préfiguration d'un observatoire lyonnais du climat
}

\author{
Didier Soto, Florent Renard et Emmanuel Thimonier-Rouzet
}

\section{NOTE DE L'AUTEUR}

Ce travail a été réalisé grâce au soutien financier du LabEx IMU (ANR-10-LABX-0088) de l'Université de Lyon, dans le cadre du programme « Investissements d'Avenir » (ANR-11-IDEX-0007) géré par l'Agence Nationale de la Recherche (ANR).

Les auteurs tiennent à remercier l'ensemble des membres du comité de pilotage du projet d'Epoc pour leurs précieux conseils.

\section{Introduction}

1 Les décisions prises à la suite du Grenelle Environnement, en 2007, ont porté, notamment, sur le développement des systèmes d'observation structurés et pérennes, inscrits dans un cadre pluridisciplinaire, capables de fédérer les efforts de recherche, de capitaliser les données et les expériences, et de favoriser les échanges. Consécutivement ont vu le jour un certain nombre de structures d'observation, dont les thématiques portent, entre autres, sur les impacts du changement climatique en milieu urbain.

Le changement climatique constitue une problématique qui se prête à l'observation, de par l'instrumentation, la nécessaire pluridisciplinarité et la dimension prospective qu'appellent sa mesure et son analyse. Aujourd'hui, son étude n'est plus seulement l'apanage des climatologues; elle est déclinée dans des champs disciplinaires divers comme l'architecture, l'urbanisme (Desjardins, 2011 ; Colombert et al., 2012), la santé environnementale (INVS, 2010), l'énergétique, l'économie, la psychologie (Van der 
Linden, 2015), ou bien la gestion de l'eau en milieu urbain, à l'instar de ce que réalisent déjà certains observatoires comme l'observatoire de terrain en hydrologie urbaine (Othu) ou bien l'Observatoire nantais des environnements urbains (Onevu). Elle intéresse, non plus seulement la communauté des chercheurs, mais également les techniciens des collectivités territoriales, les consultants (Chemin et Thimonier-Rouzet, 2010), les réseaux associatifs et les dirigeants d'entreprise (EPE et Onerc, 2014). L'actualité récente autour de la $21^{\text {ème }}$ Conférence des Parties (Cop21) montre, également, la place occupée par la démocratie participative ${ }^{1}$, qui implique un meilleur accès à l'information environnementale et une participation des citoyens au processus décisionnel.

3 Le changement climatique est également considéré, aujourd'hui, comme un métarisque, dans le sens où il contribue à amplifier, directement, les aléas (vagues de chaleur, inondations, etc.) à l'origine des risques sanitaires, économiques ou environnementaux et, indirectement, leurs conséquences sur des enjeux plus ou moins vulnérables et des territoires plus ou moins résilients. L'exemple de la canicule de 2003, qui a provoqué une surmortalité inhabituelle de 15000 personnes sur le territoire français (Quenault, 2013a), est révélateur des impacts d'une vague de chaleur de longue durée, accompagnée d'un épisode de pollution à l'ozone, qui a touché, en premier lieu, des individus vulnérables, tels que les personnes âgées vivant en milieu urbain (Besancenot, 2007). Les villes apparaissent, ainsi, de plus en plus nettement, comme des territoires de risque, non seulement à cause de leurs fortes vulnérabilités, mais aussi parce que leur capacité de réponse est trop souvent insuffisante (Quenault, 2013b).

4 L'objectif des politiques publiques d'aménagement du territoire consiste donc à diminuer les risques futurs auxquels seront soumis les sociétés et leur environnement, au travers d'actions diminuant les impacts effectifs du changement climatique et améliorant la capacité de réponse des enjeux concernés. Le premier cas de figure relève d'une stratégie d'atténuation. L'attention est portée sur la réduction de l'intensité et de la fréquence de l'aléa, en réduisant l'accroissement global des températures via, notamment, la réduction des émissions de gaz à effet de serre (GES - Onerc, 2010; Quenault, 2013a). En revanche, agir sur la capacité de réponse des enjeux nécessite de prendre en compte leur vulnérabilité et leur résilience, et relève, dans ce second cas de figure, d'une stratégie d'adaptation.

5 La déclinaison des stratégies institutionnelles nationales d'atténuation et d'adaptation se fait, aujourd'hui, à plusieurs échelles de territoires, par l'intermédiaire des Schémas Régionaux Climat, Air Énergie (SRCAE) et, notamment, plus localement, des Plans Climat Énergie Territoriaux (PCET), obligatoires pour les collectivités de plus de 50000 habitants (article L.229-26 du Code de l'Environnement).

6 Cette étude s'inscrit, logiquement, dans cette approche pluridisciplinaire et territoriale du changement climatique et présente les conditions d'établissement d'un observatoire du climat sur le territoire métropolitain lyonnais ${ }^{2}$. Lancé depuis 2005, le PCET de la Métropole de Lyon a, d'abord, initié sa stratégie d'atténuation par la conduite de 26 actions sectorielles (habitat, entreprises, énergie, déplacements) et transversales, pour tenter de parvenir aux objectifs des « $3 \times 20$ » à l'horizon 2020 et du Facteur 4 à l'horizon 2050. Une des particularités de cette politique est d'avoir envisagé une démarche partenariale avec les différents acteurs du territoire sur la manière d'atteindre ces objectifs, par le biais, notamment, des Conférences Énergie Climat (Grand Lyon, 2010). Dans l'animation du volet « adaptation » de son PCET, la Métropole 
de Lyon souhaiterait reproduire la même démarche et s'est rapprochée de la communauté locale des chercheurs, réunis au sein du Laboratoire d'Excellence Intelligences des mondes urbains (LabEx Imu), de l'Agence d'urbanisme de l'aire métropolitaine lyonnaise et de Météo-France, pour conduire une étude de préfiguration d'un observatoire local du climat (projet Epoc) et des effets du changement climatique. Ce projet a débuté en 2014 et ce texte en présente le premier point d'avancement. Deux points sont présentés ci-après: le premier expose la nécessité de créer un réseau d'observation, de diffusion, de suivi et de modélisation des effets locaux du changement climatique; le second propose une réflexion prospective sur les missions potentielles d'un tel observatoire.

\section{Un observatoire pour accompagner la politique institutionnelle d'adaptation au changement climatique : une idée légitime}

7 L'idée d'un observatoire local du climat a été motivée par le constat suivant : l'étude des caractéristiques du climat urbain, ainsi que l'analyse des conséquences locales du changement climatique font, aujourd'hui, l'objet d'initiatives porteuses mais insuffisamment connectées, qui nécessitent une structure d'interface pluridisciplinaire capable de les fédérer.

\section{Des initiatives porteuses à connecter}

8 Un des verrous identifiés à l'échelle locale par les gestionnaires porte, actuellement, sur une meilleure caractérisation du climat urbain, indispensable pour mieux comprendre les impacts potentiels du changement climatique. L'effet d'îlot de Chaleur Urbain (ICU), qui se caractérise par un différentiel de températures entre une agglomération et ses alentours moins urbanisés (Oke, 1973 ; Landsberg, 1981 ; Kim et Guldmann, 2014, entre autres) peut, ainsi, dans la perspective du changement global, amplifier les aléas climatiques prévus à l'échelle régionale (hausse de la température moyenne annuelle, augmentation du nombre de jours de fortes chaleurs - Jouzel et al., 2014). Des travaux ont donc été menés, en ce qui concerne le territoire lyonnais, pour mieux identifier et caractériser les ICU (Champiat, 2009; Agence d'urbanisme pour le développement de l'agglomération lyonnaise, 2010 ; Diallo-Dudek, 2015) et formuler des préconisations ou présenter des expérimentations pour atténuer leurs effets (Maillard et al., 2014). Cependant, ils concernent différentes catégories d'acteurs du territoire (chercheurs, techniciens de collectivités, dirigeants d'entreprises), qui ne partagent pas, encore, une culture commune de mutualisation des compétences locales. Cependant, tous présentent le même objectif de pouvoir développer des outils pour instrumenter et modéliser le climat de l'agglomération lyonnaise.

9 Par ailleurs, les diagnostics Climat, réalisés par la Ville de Lyon et la Métropole de Lyon (encore dans leur version provisoire à l'heure de la parution de ce texte), et établis selon le modèle de l'Ademe (2012), apportent un éclairage global sur l'ensemble des enjeux potentiellement impactés par les effets du changement climatique. Cependant, ces diagnostics n'adoptent pas une approche spatialisée, focalisée sur la ville, et ne permettent ni de procéder à une évaluation des vulnérabilités territoriales, ni de parvenir, de facto, à une approche globale des risques par combinaison de l'aléa, de la 
vulnérabilité des enjeux et de la résilience territoriale. Les gestionnaires sont, aujourd'hui, confrontés à ce verrou scientifique et ne savent pas comment prioriser l'action territoriale permettant de réduire les vulnérabilités en cas de survenue de fortes chaleurs.

Une des solutions pour permettre de se focaliser sur le territoire, véritable trait d'union entre tous les acteurs de l'adaptation, pourrait être une structure opérationnelle pluridisciplinaire, qui puisse contribuer à rapprocher les chercheurs, dépositaires des savoirs académiques, les praticiens, porteurs des connaissances de l'action quotidienne, et la société civile, qui est amenée à mieux développer ses capacités adaptatives. Un projet d'observatoire, de par sa capacité générale à produire de la donnée pérenne, de la diffuser, et de l'analyser semble idoine. Si des structures d'observation existent déjà au sein de la région Rhône-Alpes, aucune ne permet, comme nous allons le voir cidessous, de couvrir les problématiques de recherche en lien avec la mesure du climat urbain, ou bien l'adaptation locale aux effets du changement climatique.

\section{Les structures d'observation déjà présentes sur le territoire}

11 Les observatoires, qui prennent en compte les effets du changement climatique en milieu urbain, revêtent, aujourd'hui, plusieurs formes et résultent, généralement, soit d'un rapprochement de structures universitaires de recherche, soit d'un pilotage par des collectivités territoriales et des établissements publics ${ }^{4}$. Comprendre cette distinction entre une catégorie d'observatoires à visée scientifique et une autre à visée davantage socio-économique, est nécessaire, de manière à pouvoir déterminer le positionnement de la structure envisagée et ses missions : s'agit-il de créer de la donnée pour alimenter des recherches académiques, de compiler des données existantes pour une utilisation par des gestionnaires, ou bien les deux? Cette question est fondamentale car les moyens financiers à engager ne seront pas forcément les mêmes selon la réponse.

Les objectifs de la structure envisagée ne doivent, également, pas être redondants avec ceux des observatoires qui couvrent le territoire d'étude, parmi lesquels :

- l'Observatoire régional des effets du changement climatique (Orecc), qui constitue un observatoire à visée socio-économique sur le territoire de la région Rhône-Alpes ;

- l'Observatoire régional de l'énergie et des gaz à effet de serre (Oreges), qui constitue un outil pour accompagner la politique régionale de réduction des émissions de gaz à effet de serre ;

- Air Rhône-Alpes, association dont l'objectif est de mesurer et d'analyser la qualité de l'air.

13 À l'échelle locale, c'est-à-dire celle qui couvre exclusivement le territoire métropolitain lyonnais, le seul observatoire en lien avec le climat urbain est l'othu, qui se présente sous la forme d'une Fédération de Recherche, comme l'Onevu. Ses activités d'observation et de recherche ont pour objectifs de mesurer et de modéliser les flux d'eau ainsi que l'efficacité des systèmes de gestion des eaux, dans une optique d'améliorer les pratiques courantes.

14 Ainsi, compte-tenu de la volonté des observatoires régionaux de ne pas substituer aux politiques publiques locales, et compte-tenu de la spécificité thématique de l'Othu, l'idée de créer un observatoire local du climat et des effets du changement climatique est légitime. 


\section{Quel observatoire pour quelles thématiques?}

15 Si l'idée d'un observatoire local du climat paraît ainsi opportune, il convient désormais de s'interroger sur le type d'observatoire souhaité pour accompagner la politique institutionnelle d'adaptation au changement climatique. Compte-tenu de la dimension territoriale et pluridisciplinaire de la problématique, une structure d'interface est à privilégier, de manière à concerter les compétences locales et à créer un dialogue partenarial. Une réflexion prospective est également proposée sur les réalisations potentielles et les attendus d'un tel observatoire.

\section{Une structure d'interface}

De manière à s'inscrire dans une politique locale d'adaptation qui puisse adopter une approche globale des risques d'origine climatique, l'observatoire envisagé doit constituer une interface entre les structures d'enseignement supérieur et de recherche, la société civile, ses gestionnaires territoriaux, ses élus, ainsi que les entreprises. L'observatoire doit lier ces différentes polarités et devenir un outil de traduction à la fois des problématiques de recherche, dans un ancrage pluridisciplinaire, mais aussi des besoins des gestionnaires et des entrepreneurs. Il doit permettre d'ancrer la conscience du changement climatique dans l'esprit des décideurs et des citoyens et permettre, ainsi, d'éviter des erreurs de mal-adaptation ${ }^{5}$. L'idée n'est pas tant de se placer, uniquement, en amont (production de données) ou en aval (aide à la décision) en termes de production de connaissances et de mutualisation des compétences, mais de faire interagir les sphères qui composent le système territorial, de manière à définir une stratégie commune et concertée d'adaptation au changement climatique.

Dans le cadre du projet Epoc, une consultation a été initiée dans le but de détecter les partenaires potentiels de l'observatoire. Il en ressort, comme mentionné en introduction, que la communauté locale des chercheurs, qui s'intéresse à la problématique du changement climatique, est très diversifiée. Elle produit aujourd'hui des savoirs issus à la fois des sciences humaines et sociales, des sciences de l'ingénieur, des sciences biologiques, et des sciences médicales et sanitaires. De même, la communauté praticienne est également segmentée selon les établissements concernés (la Métropole de Lyon, l'Agence d'urbanisme de l'aire métropolitaine lyonnaise, MétéoFrance, l'Agence Régionale de la Santé), qui s'intéressent à la question. Dans le cadre de cette consultation, tous ont manifesté l'intérêt de dépasser les limites propres de leurs disciplines ou de leurs métiers pour créer une connaissance pluridisciplinaire des effets du changement climatique. L'observatoire doit donc reposer sur ce socle de chercheurs et de praticiens, dont le partenariat, aujourd'hui incontournable, doit contribuer à briser les frontières longtemps érigées entre savoirs et action (Chemin et ThimonierRouzet, 2010). À ce socle doit également s'adjoindre le monde de l'entreprise, investi à l'échelle locale dans cette problématique, et la société civile par le biais, notamment, du tissu associatif.

Les conditions d'un dialogue entre chacune de ces polarités sont, aujourd'hui, facilitées par le travail et la vision pluridisciplinaires sur les mondes urbains du LabEx Imu. Cependant, si les acteurs rencontrés convergent vers un intérêt commun, des divergences subsistent encore entre la production des savoirs académiques, leur interprétation et leur transposition opérationnelle, si bien que, dans le cadre de la 
problématique de l'adaptation au changement climatique, des questions fondamentales doivent encore être résolues : à quelles conditions climatiques doit-on concrètement s'adapter? Quels visages (forme, couleur, matériaux) doit avoir la ville de demain? Comment peuvent être améliorées les capacités adaptatives institutionnelles et civiles? De quelle manière est-il possible d'évaluer, et par là même, de réduire la vulnérabilité des enjeux humains et environnementaux?

Par ailleurs, les échanges avec les chercheurs et les praticiens ont révélé la nécessité de disposer d'un langage commun sur des concepts forts en lien avec l'adaptation comme celui de "vulnérabilité » ou bien celui de « résilience », dont la polysémie alimente des interprétations ambigües voire contradictoires, comme l'ont déjà constaté DjamentTran et Reghezza-Zitt (2012), ainsi que Quenault (2013b). D'un point de vue plus large, la confusion même entre stratégies d'atténuation et d'adaptation tend à privilégier la question énergétique dans les politiques publiques consacrées à la ville durable, comme le souligne Kergomard (2015). Il incombe notamment aux chercheurs locaux rassemblés au sein de l'observatoire lyonnais du climat, de jouer un rôle de traducteur auprès des décideurs, des gestionnaires et des dirigeants d'entreprises, afin de partager la complexité des réflexions portées par les sciences humaines et sociales autour des concepts de vulnérabilité territoriale et de résilience urbaine, dans un contexte de changement climatique global (Brooks et al., 2005 ; Adger, 2009 ; Posey, 2009).

\section{Proposition de quelques thématiques potentielles de l'observatoire}

Les interrogations, mentionnées ci-dessus, ont été à l'origine d'un questionnement autour des thématiques potentielles de l'observatoire. Les besoins des gestionnaires, mais aussi les intérêts manifestés par les premiers participants, font ressortir essentiellement trois verrous scientifiques: la mesure et la modélisation du climat local, l'évaluation de la vulnérabilité urbaine et le diagnostic de résilience territoriale (illustration 1). 
Illustration 1 - Schéma organisationnel des principales thématiques de recherche en lien avec le projet Epoc

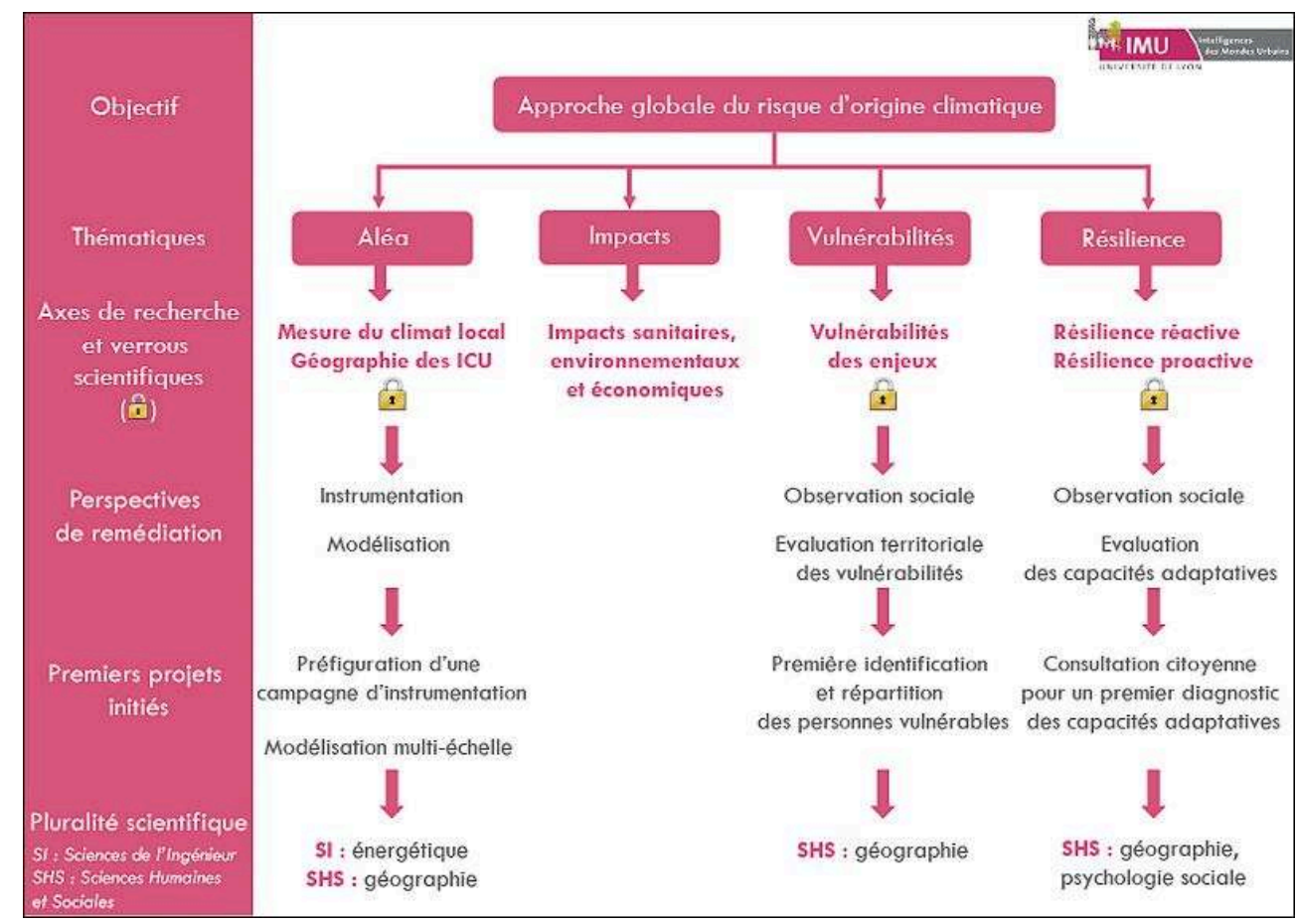

\section{Une meilleure caractérisation du risque de fortes chaleurs en milieu urbain}

Dans la perspective du changement climatique (Météo-France, 2011 ; Jouzel et al, 2014), la hausse de la moyenne annuelle de la température de l'air (comprise entre 0,6 et $1,3^{\circ} \mathrm{C}$ à l'horizon $2050^{6}$, par rapport à la période 1976-2005) et l'augmentation du nombre de jours de fortes chaleurs en été (entre 0 et 5 jours), potentiellement amplifiées par le milieu urbain avec l'effet d'ICU, contribuent à amplifier les aléas auxquels sont soumis les enjeux vulnérables du territoire (cf. chapitre suivant). La caractérisation du climat urbain et des effets potentiels de son changement est donc primordiale dans la déclinaison de la stratégie d'adaptation du territoire, de manière à réduire les impacts sanitaires et environnementaux d'une future vague de chaleur d'ampleur exceptionnelle.

Cependant, les relevés météorologiques pérennes sur le territoire urbain lyonnais proviennent de la seule station de Lyon-Bron, située en dehors du centre urbain dense et soumise à une périurbanisation intense ces cinquante dernières années (illustration 2). Dans un périmètre plus élargi que le territoire institutionnel de la Métropole de Lyon, à savoir l'unité urbaine de Lyon, selon le zonage Insee, il existe également quelques stations météorologiques permettant un enregistrement automatique sur lesquelles s'appuyer, telle la station météorologique synoptique ${ }^{7}$ de Lyon Saint-Exupéry, à proximité de l'aéroport international. Cependant, le constat est flagrant : le centre urbain dense est dépourvu de stations météorologiques autorisant une acquisition automatique et expertisée de données de températures de l'air, selon une fréquence horaire minimale. 
Illustration 2 - Réseau des stations météorologiques de Météo-France sur l'unité urbaine de Lyon

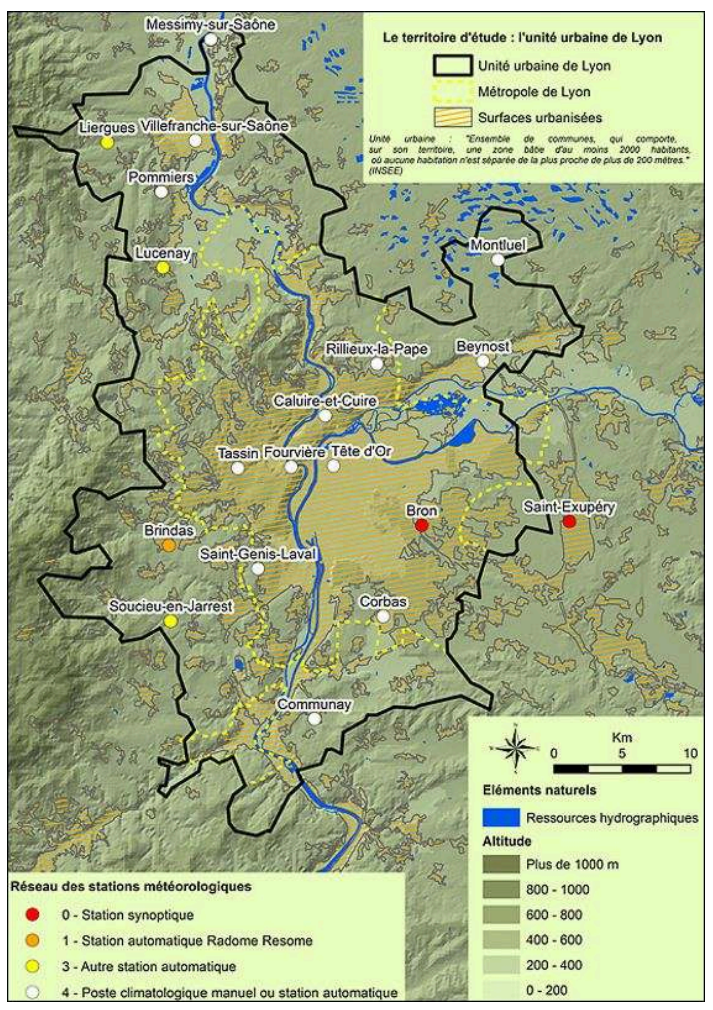

23 Une des missions de l'observatoire peut donc être de contribuer à résoudre ce problème de désert métrologique, de manière à permettre, notamment, une meilleure géographie des ICU. Pour ce faire, trois méthodes apparaissent possibles et complémentaires.

La première est de développer un réseau de capteurs, afin de pouvoir instrumenter des secteurs pour lesquels l'effet d'îlot de chaleur urbain est susceptible d'être récurrent. Deux approches sont actuellement discutées par les décideurs du projet Epoc: une approche quantitative, qui consisterait à développer un réseau de stations multiparamètres (températures, précipitations, pression, vitesse et direction du vent, humidité relative), permettant une acquisition automatique des données pouvant être expertisées dans un temps différé le plus court possible ; une approche qualitative, qui consisterait à équiper des observateurs bénévoles, susceptibles de contribuer à une acquisition manuelle ou semi-automatique des données, tandis que l'expertise des données serait différée dans un temps plus long. Chaque approche présente des avantages et des inconvénients : le coût des stations multi-paramètres ne permet pas d'assurer une couverture fine $\mathrm{du}$ territoire d'étude, tandis que le recours à des observateurs bénévoles, moins coûteux, ne permet pas de garantir pleinement une donnée fiable.

La seconde méthode est de s'appuyer sur l'imagerie satellitaire, immédiatement disponible et peu onéreuse au regard de l'instrumentation de terrain, mais pouvant présenter des difficultés d'exploitation suite, notamment, à la faible probabilité de disposer d'une image nette exploitable.

Enfin, la troisième repose, davantage, sur la modélisation numérique. Des modèles météorologiques de type méso-échelle, tel que Meso- $\mathrm{NH}^{8}$ permettant une approche multi-échelles, couplés à des modèles urbains globaux, tel que TEB $^{9}$ (Town Energy Balance), permettent déjà de simuler la ville et le processus d'îlot de chaleur. Une 
première modélisation de l'îlot de chaleur urbain lyonnais a été proposée avec l'outil Meso-NH (Diallo-Dudek, 2015). Lors des journées étudiées, des gradients de température très resserrés apparaissent, notamment, dans l'Est lyonnais, mettant en évidence des micros ICU et IFU (Îlots de Fraîcheur Urbain). Cependant, afin d'évaluer plus précisément les microclimats locaux et les conditions thermiques intérieures, il est nécessaire de modéliser le comportement énergétique du bâtiment et, plus particulièrement, les effets de l'inertie thermique, responsable du stockage de chaleur dans les structures et du maintien de températures élevées en soirée. Une approche statistique, basée sur la modélisation, est également nécessaire afin de fiabiliser l'approche.

27 Pour lever ce verrou scientifique, un travail universitaire a été conduit, dans le cadre du projet Epoc, sur la faisabilité d'une campagne d'instrumentation (illustration 1) et porte sur le recensement du matériel existant et l'analyse du coût et du positionnement des futurs capteurs. Par ailleurs, un autre projet de recherche a débuté, en novembre 2015, sur des perspectives de modélisation multi-échelle, de celle de la ville à celle du quartier.

28 À terme, une des orientations de l'observatoire pourrait être de garantir le relevé pérenne des mesures en milieu urbain dense et de contribuer à l'analyse des données instrumentales, comme le fait déjà l'Othu, en ce qui concerne les relevés pluviométriques. Ce travail est indispensable pour valider les sorties de modèle qui, en l'absence de données météorologiques du centre urbain dense statistiquement significatives sur le temps long, constitueront, dans l'immédiat, le principal support d'aide à la décision pour le gestionnaire.

\section{Une évaluation territoriale de la vulnérabilité urbaine à construire}

29 En termes de vulnérabilité urbaine aux aléas potentiellement amplifiés par le changement climatique, il est désormais connu que si on se focalise sur l'aléa " fortes chaleurs » en milieu urbain, les personnes âgées, les enfants, les publics vulnérables (handicapés, malades chroniques), les individus à habitat précaire, à revenus très faibles ou souffrant d'isolement social, sont les plus exposées (Besancenot, 2002,2007; Onerc, 2007 ; INVS, 2010 ; Quenault, 2013b ; EHESP, 2014). Les personnes âgées, par exemple, sont généralement fragilisées par la plus faible capacité de récupération de leur organisme, notamment pendant la nuit, et par leur plus forte médicalisation, qui altère leur capacité de récupération thermique. Cette problématique est d'autant plus à prendre en compte que la population générale de l'agglomération vieillit : la part des personnes âgées de plus de 85 ans augmentera de $80 \%$ à l'horizon 2030 (Grand Lyon, 2008). Par ailleurs, environ $19 \%$ des ménages l'agglomération lyonnaise sont caractérisés par une précarité énergétique ${ }^{10}$, selon les diagnostics climat institutionnels. De plus, l'accentuation probable des effets causés par la pollution, particulièrement pendant les épisodes de vagues de chaleur, par l'augmentation de la durée de la saison pollinique, ou bien par l'extension des aires de répartition des maladies transmises par un vecteur vivant (moustique tigre, par exemple), implique également une exposition accrue des populations les plus fragiles.

30 En revanche, les diagnostics climats institutionnels ne permettent pas de procéder à une évaluation territoriale opérationnelle de la vulnérabilité humaine et environnementale à l'aléa « vague de chaleur ». Ainsi, un premier travail est à conduire pour procéder à une évaluation fine des vulnérabilités territoriales, à partir de 
méthodes d'analyse multicritère qui s'appuient sur des jugements d'experts, comme cela a été fait, sur le territoire métropolitain lyonnais, par Renard et Soto (2015) et Soto et Renard (2015), en ce qui concerne les risques d'inondation et d'origine technologique. Pour pouvoir évaluer la vulnérabilité et tenter de la réduire, il est donc nécessaire de capitaliser les informations contenues dans les deux diagnostics institutionnels et de procéder à une évaluation exhaustive de la vulnérabilité territoriale des enjeux.

31 C'est l'une des raisons pour lesquelles l'observatoire pourrait être saisi ; celui-ci solliciterait les compétences locales identifiées pour constituer le panel d'experts constitués. La cartographie entreprise permettrait alors d'identifier des îlots de vulnérabilité qui, s'ils venaient à coïncider avec des quartiers au sein desquels se forment régulièrement des micros ICU, constitueraient des secteurs prioritaires à aménager (végétalisation, amélioration du confort thermique) et à sensibiliser (campagnes de prévention, actions participatives sur le terrain). Un suivi, sous la forme d'une veille, pourrait alors être envisagé pour assurer une observation sociale pérenne, comme le font déjà certains observatoires sociaux et sanitaires. Un travail universitaire, toujours dans la continuité du projet Epoc, a d'ailleurs été mené sur ce sujet en ce qui concerne le territoire lyonnais, dans le but de de procéder à une première identification et répartition des personnes les plus vulnérables sur le territoire d'étude (illustration 1).

\section{Une meilleure prise en compte des capacités adaptatives}

32 Une autre approche peut être conduite pour appréhender la vulnérabilité liée, notamment, à la résilience des systèmes urbains : il s'agit de déterminer la capacité de réponse des individus et des groupes sociaux aux aléas climatiques. En effet, si la résilience d'un système est, en partie, due à sa capacité d'absorption face à une perturbation et à son auto-organisation, il convient aussi de s'intéresser à son aptitude à construire et à accroître sa capacité adaptative et sa capacité d'apprentissage (Folke et al., 2002), conditionnée, notamment, par l'accès aux savoirs et aux représentations de la population (Espon Climate, 2011). Par le biais de cette démarche, il s'agit également de valoriser la démocratie participative et d'impliquer le citoyen dans l'accès, la production et la diffusion de l'information. Les recherches participatives, de par leur capacité à faire émerger et confronter les connaissances d'usage et les savoirs académiques, semblent constituer un moyen approprié pour évaluer les connaissances et les représentations des individus. Cette approche pourrait être l'une des missions potentielles de l'observatoire, permettant de réduire la vulnérabilité et d'augmenter la résilience sociale, notamment dans sa dimension proactive.

33 À ce sujet, a été conduite, sur le territoire lyonnais, une recherche participative sur les perceptions des citoyens aux effets du changement climatique et leurs savoir-faire en cas de survenue d'un phénomène remarquable (les fortes chaleurs sont à nouveau prises comme sujet d'étude). Cette recherche a été conduite par le service de médiation scientifique de l'Université de Lyon (service Sciences et Société), en lien avec une équipe pluridisciplinaire locale de chercheurs en sciences humaines et sociales (géographes, psychologues sociaux, entre autres). Les premiers résultats de cette consultation sont, courant novembre 2015, en cours d'analyse.

34 Ainsi, même si l'observatoire est en phase de concrétisation opérationnelle fin 2015, l'intérêt du projet Epoc aura été d'avoir permis d'incuber trois projets de recherche sur 
des thématiques qui, à court terme, permettront d'apporter des réponses aux questionnements liées à la conduite d'une stratégie institutionnelle d'adaptation. À moyen terme, ils pourraient donner lieu à une orientation selon des axes thématiques (illustration 1), à condition toutefois de ne pas cloisonner la réflexion qui doit se positionner dans une perspective d'interface professionnelle et de croisement pluridisciplinaire.

\section{Conclusion} structure. L'architecture et la gouvernance de l'observatoire n'ont pas encore été décidées. Elles ne peuvent l'être tant que ne se seront pas déclarés les principaux animateurs de la structure. Si les rencontres bilatérales, menées lors de la consultation des acteurs territoriaux, ont permis d'éveiller une curiosité pour le projet, il est nécessaire, désormais, de créer des moments de rencontre «hybride " (journées de concertation, manifestations scientifiques et techniques, formations professionnelles), $\mathrm{au}$ cours desquels les partenaires potentiels échangent et construisent des connaissances à partir de leurs intérêts scientifique, technique, économique ou politique. Par ailleurs, l'interaction avec les structures d'observation locales et régionales doit être clairement définie, de manière à ce qu'elles puissent également 
jouer un rôle partenarial ou de commanditaire. Ces conditions apparaissent comme des prérequis sans lesquelles il parait difficile d'envisager la question du financement des moyens humains et matériels nécessaires pour rendre opérationnel l'observatoire lyonnais du climat.

\section{BIBLIOGRAPHIE}

Ademe, 2012. Diagnostic de vulnérabilité d'un territoire au changement climatique. 53 p.

Adger W.N., Dessai S., Goulden M., Hulme M., Lorenzoni I., Nelson D.R., Naess L.O., Wolf J., Wreford A., 2009. Are there social limits to adaptation to climate change ? Climatic change, vol. 93 , $\mathrm{n}^{\circ} 2$, p. 335-354.

Agence d'urbanisme pour le développement de l'agglomération lyonnaise, 2010. Identification des îlots de chaleur de l'agglomération lyonnaise, vulnérabilité des populations et mesures envisageables de résorption. $98 \mathrm{p}$.

Besancenot J.-P., 2002. Vagues de chaleur et mortalité dans les grandes agglomérations urbaines. Environnement Risques Santé, vol. 1, p. 229-240.

Besancenot, J.-P., 2007. Notre santé à l'épreuve du changement climatique. Paris, Delachaux et Niestlé, $221 \mathrm{p}$.

Brooks N., Adger W.N., Kelly M.P., 2005. The determinants of vulnerability and adaptive capacity at the national level and the implications for adaptation. Global Environmental Change, vol. 15, p. 151-163.

Champiat C., 2009. Identifier les ICU pour réduire l'impact sanitaire des vagues de chaleur. Environnement Risques Santé, vol. 8, p. 399-411.

Chemin C., Thimonier-Rouzet E., 2010. Le rapprochement Chercheur-Consultant en aménagement du territoire: un apport à haute valeur ajoutée ? EchoGéo [En ligne], Sur le Vif, mis en ligne le 18 février 2010. URL : http://echogeo.revues.org/11635; DOI : 10.4000/echogeo.11635

Colombert M., Salagnac J.-L., Morand D., Diab Y., 2012. Le climat et la ville : la nécessité d'une recherche croisant les disciplines. Vertigo - la revue électronique en sciences de l'environnement [En ligne], Hors-série 12 | mai 2012, mis en ligne le 04 mai. URL : http://vertigo.revues.org/11811 ; DOI : $10.4000 /$ vertigo. 11811

Desjardins X., 2011. Pour l'atténuation du changement climatique, quelle est la contribution possible de l'aménagement du territoire. Cybergeo : European Journal of Geography [En ligne], Aménagement, Urbanisme, document 523, mis en ligne le 22 mars 2011. URL : http:// cybergeo.revues.org/23531; DOI : 10.4000/cybergeo.23531

Diallo-Dudek J., 2015. Land Surface Temperature in the urban area of Lyon metropolis: a comparative study of remote sensing data and MesoNH model simulation. Actes du colloque Joint Urban Remote Sensing Event, Lausanne.

Djament-Tran G., Reghezza-Zitt M., 2012. Résiliences urbaines : Les villes face aux catastrophes. Paris, Le Manuscrit, $364 \mathrm{p}$. 
EHESP, 2014. Guide - Agir pour un urbanisme favorable à la santé. $192 \mathrm{p}$.

EPE, Onerc, 2014. Les entreprises et l'adaptation au changement climatique. 56 p.

Espon Climate, 2011. Climate Change and Territorial Effects on Regions and Local Economies. 264 p.

Folke C., Carpenter S., Elmqvist T., Gunderson L., Holling C.S., Walker B., 2002. Resilience and Sustainable Development: Building Adaptive Capacity in a World of Transformations. Ambio, vol. $31, n^{\circ}$ 5, p. 437-440.

Grand Lyon, 2010. Conférence Energie Climat - Co-construire l'avenir de l'agglomération. 12 p.

Grand Lyon, 2008. Ville, santé et réchauffement climatique.48 p.

INVS, 2010. Impacts sanitaires du changement climatique en France. $57 \mathrm{p}$.

Jouzel J., Ouzeau G., Deque M., Jouini M., Planton S., Vautard R., 2014. Le climat de la France au XXIème siècle. Volume 4. Scénarios régionalisés : édition 2014 pour la métropole et les régions d'outre-mer.64 p.

Kergomard C., 2015. Résilience urbaine et changement climatique global. in Reghezza-Zitt M. et Rufat S, Résiliences - Sociétés et territoires face à l'incertitude, aux risques et aux catastrophes. Londres, Iste Editions, p. 123-140.

Kim J.-P, Guldmann J.-M, 2014. Land-use planning and the urban heat island. Environment and Planning B: Planning and Design, vol. 41, p. 1077-1099.

Landsberg H.E, 1981. The Urban Climate. New-York, Academic Press, 289 p.

Maillard P., David F., Dechesne M., Bailly J.B, Lesueur E., 2014. Caractérisation des îlots de chaleur urbains et test d'une solution d'humidification de chaussée dans le quartier de la Part-Dieu à Lyon. Techniques Sciences Méthodes, nº 6, p. 23-35.

Météo-France, 2011. Étude du changement climatique pour le SRCAE Rhône-Alpes.73 p.

Oke T.R, 1973. City size and the urban heat island. Atmospheric Environment, vol. 7, p. 769-779.

Onerc, 2007. Changement climatique et risques sanitaires en France.208 p.

Onerc, 2010. Villes et adaptation au changement climatique.158 p.

Posey J., 2009. The determinants of vulnerability and adaptive capacity at the municipal level: evidence from floodplain management programs in the United States. Global Environmental Change, vol. 19, num. 4, p. 482-493.

Quenault B., 2013a. La ville durable au défi du couple vulnérabilité/adaptation au changement climatique. In Bertrand F. et Rocher L., Les territoires face aux changements climatiques. Une première génération d'initiatives locales. Bruxelles, P.I.E. Peter Lang, p. 175-202.

Quenault B., 2013b. Retour critique sur la mobilisation du concept de résilience en lien avec l'adaptation des systèmes urbains au changement climatique, EchoGéo [En ligne], 24 | 2013, mis en ligne le 10 juillet 2013. URL : http://echogeo.revues.org/13403 ; DOI : 10.4000/echogeo.13403

Rocher L., 2013. Le climat, nouvel objet de l'action urbaine lyonnaise. In Bertrand F. et Rocher L., Les territoires face aux changements climatiques. Une première génération d'initiatives locales. Bruxelles, P.I.E. Peter Lang, p. 125-150.

Renard F., Soto D., 2015. Une représentation du risque à l'intersection de l'aléa et de la vulnérabilité : cartographies des inondations lyonnaises. Geographica Helvetica, vol. 70, p. 333-348.

Soto D., Renard F., 2015. New prospects for the spatialisation of technological risks by combining hazard and the vulnerability of the assets. Natural Hazards, vol. 79 (3), p. 1531-1548. 
Van der Linden S., 2015. The social-psychological determinants of climate change risk perceptions: Towards a comprehensive model. Journal of Environmental Psychology, vol. 41, p. $112-124$

\section{NOTES}

1. Site du réseau du Ministère de l'Écologie, du Développement Durable et de l'Énergie : http:// www.developpement-durable.gouv.fr/Je-m-informe,42332.html (consulté le 20 avril 2015).

2. Le territoire métropolitain lyonnais concerne celui de la collectivité territoriale de la Métropole de Lyon, formée le 1er janvier 2015 et composée de 59 communes pour une population de près de 1,3 million d'habitants.

3. L'expression « 3x20 » désigne l'objectif européen de réduire de $20 \%$ les émissions de gaz à effet de serre (GES), d'améliorer de $20 \%$ l'efficacité énergétique et de porter à $20 \%$ la part des énergies renouvelables dans la consommation finale d'énergie. Le Facteur 4 désigne l'objectif national de réduire par quatre les émissions territoriales de GES à l'horizon 2050 (Rocher, 2013).

4. Sans procéder à un recensement exhaustif des observatoires existants, il est possible d'illustrer la différence entre ces deux types de structure en comparant l'Onevu et l'Observatoire régional des effets du changement climatique (Orecc). Le premier est administrativement rattaché à l'Institut de Recherche en Sciences et Techniques de la Ville (IRSTV), qui est une Fédération de Recherche, tandis que le second est piloté par une direction multipartite, au sein de laquelle se retrouvent l'État, la Région Rhône-Alpes et l'Ademe. L'Onevu est caractéristique des observatoires impulsés par le monde de la Recherche dans le sens où une des priorités est donnée à la création et à l'analyse de mesures nouvelles, notamment météorologiques, tandis que les missions de l'Orecc portent sur la compilation de données existantes pour créer des indicateurs applicables à une échelle intra - régionale, comme le font d'autres observatoires similaires (l'Observatoire Climat en Nord-Pas de Calais - OCNPC ou l'Observatoire régional énergie changement climatique air en Aquitaine - Orecca).

5. Mal adaptation : action menée pour faire face au changement climatique, mais qui contribue, de manière non-intentionnelle, à augmenter la vulnérabilité au lieu de la réduire. Un exemple de mal-adaptation peut être le recours massif à des outils de climatisation pour rafraîchir l'air intérieur au lieu d'investir dans l'isolation thermique des bâtiments (Ministère de l'Écologie, du Développement Durable et de l'Énergie: http://www.developpement-durable.gouv.fr/Leconcept-de-mal-adaptation.html - consulté le 22 avril 2015).

6. Cette valeur a été choisie car elle correspond, peu ou prou, à celle retenue dans les documents de planification urbaine comme le Scot 2030 de l'agglomération lyonnaise.

7. Les stations synoptiques (type 0 ) sont considérées comme celles communiquant les données les plus complètes et les plus fiables, selon Météo-France (http://climatheque.meteo.fr/aide/ climatheque/reseauPostes/aideTypePoste.html). S'ensuivent les stations automatiques de type Radome-Resome (type 1), les stations automatiques non Radome-Resome (type 2), les stations automatiques à expertise différée (type 3) et les postes climatologiques manuels ou les autres stations automatiques à acquisition et expertise différées (type 4)

8. Site du modèle Meso-NH: http://mesonh.aero.obs-mip.fr/mesonh51 (consulté, pour la dernière fois, le 15/10/15)

9. Site du modèle TEB: http://www.cnrm.meteo.fr/spip.php?article199 (consulté, pour la dernière fois, le 15/10/15)

10. Selon la loi portant engagement national pour l'environnement du 12 juillet 2010 : "Est en situation de précarité énergétique [...] une personne qui éprouve dans son logement des difficultés particulières à disposer de la fourniture d'énergie nécessaire à la satisfaction de ses besoins élémentaires en raison de l'inadaptation de ses ressources ou de ses conditions d'habitat ». 


\section{RÉSUMÉS}

L'adaptation aux effets du changement climatique se prête idéalement à l'observation. À l'échelle locale, des projets porteurs ont été initiés ces dernières années de la part des chercheurs, des praticiens et des acteurs économiques. Ils nécessitent, toutefois, une structure opérationnelle pour mutualiser les connaissances acquises et les compétences diverses. Pour entreprendre cette démarche partenariale, une étude de préfiguration a été initiée, dont ce texte constitue un premier point d'avancement. Deux problématiques sont précisément évoquées : la nécessité de créer un réseau d'observations au regard des besoins des gestionnaires et des chercheurs, ainsi que les missions potentielles d'une telle structure.

Adaptation to climate change is ideal for observation. Promising projects, coming from researchers, practitioners and business partners, have been initiated earlier on the metropolitan territory. Now, they require an operational structure to gather and share knowledge and skills. To undertake this partnership approach, a prospective study has been initiated. In this paper, two issues are specifically mentioned: the establishment of this observatory in relation to the needs of practitioners and researchers, and the potential objectives of such an observatory.

\section{INDEX}

Keywords : multidisciplinary observatory, climate change, adaptation, hazard, vulnerability

Mots-clés : observatoire pluridisciplinaire, changement climatique, adaptation, aléa, vulnérabilité.

\section{AUTEURS}

\section{DIDIER SOTO}

Didier Soto, didier.soto@univ-lyon3.fr, est chercheur postdoctoral à l'Université de Lyon, rattaché au LabEx Imu (Intelligences des Mondes Urbains) et chercheur associé au sein du laboratoire Environnement Ville Société (UMR CNRS 5600). Il a publié récemment :

- Soto D., Renard F., 2015. New prospects for the spatialisation of technological risks by combining hazard and the vulnerability of the assets. Natural Hazards, vol. 79 (3), p. 1531-1548. - Renard F., Soto D., 2015. Une représentation du risque à l'intersection de l'aléa et de la vulnérabilité : cartographies des inondations lyonnaises. Geographica Helvetica, vol. 70, p. 333-348. - Soto D.., Renard F, Magnon A., 2014. Evaluating Environmental Risk to Technological Hazards, Using GIS Spatial Decision Making. Lecture Notes in Computer Science, vol. 8581, p. 15-25.

\section{FLORENT RENARD}

Florent Renard, florent.renard@univ-lyon3.fr, est maître de conférences à l'Université Jean Moulin Lyon 3 et membre du laboratoire Environnement Ville Société (UMR CNRS 5600). Il a publié récemment :

- Renard F., Soto D., 2014. Measuring Territorial Vulnerability? An Attempt of Qualification and Quantification. Lecture Notes in Computer Science, vol. 8582, p. 331-343.

- Renard F., Chapon P.M., Comby J., 2012. Assessing the accuracy of weather radar to track intense rain cells in the Greater Lyon area, France. Atmospheric Research, vol. 103, p. 4-19. 
- Renard F., Chapon P.M., 2010. Une méthode d'évaluation de la vulnérabilité urbaine appliquée à l'agglomération lyonnaise. L'espace géographique, vol. 39, nº 1, p. 35-50.

\section{EMMANUEL THIMONIER-ROUZET}

Emmanuel Thimonier-Rouzet, emmanuel.thimonier@univ-lyon3.fr, est chercheur associé au sein du laboratoire Environnement Ville Société (UMR CNRS 5600) et chargé d'études à l'Agence d'urbanisme de l'aire métropolitaine lyonnaise. Il a publié récemment :

- Thimonier-Rouzet E., 2014. La recette lyonnaise de l'aire métropolitaine. In La Vie-Le Monde, Atlas de la France et des Français. Paris, La Vie - Le Monde, p. 46-49.

- Thimonier-Rouzet E., 2012. La construction de l'espace urbain : un processus socio-économique. In Bonnet J., Aménagement et développement territorial. Paris, Ellipses, p. 75-98.

- Chemin C., Thimonier-Rouzet E., 2010. Le rapprochement consultant-chercheur en aménagement du territoire : un apport à haute valeur ajoutée. EchoGéo [En ligne], Sur le Vif, mis en ligne le 18 février 2010. URL : http://echogeo.revues.org/11635; DOI : 10.4000/echogeo.11635. 